\title{
Evaluation of aphasia in a culturally and linguistically diverse context: Practices of and challenges faced by speech-language pathologists
}

\author{
Fatimah Hani Hassan ${ }^{1}$, Pei-Whey Heng ${ }^{2}$, and Rahayu Mustaffa Kamal ${ }^{3}$ \\ ${ }^{1}$ Universiti Kebangsaan Malaysia \\ ${ }^{2}$ Affiliation not available \\ ${ }^{3}$ International Islamic University Malaysia - Kuantan Campus
}

September 16, 2020

\begin{abstract}
Rationale, aims and objectives: Cultural and linguistic diversity is a major challenge faced by speech-language pathologists (SLPs) in conducting evaluation of aphasia, especially in low-resource regions. Without proper consideration of cultural and linguistic issues when adapting English-based language tests to other languages, performance of individual with aphasia may not be valid and reliable. The present study was conducted: (a) to identify the practices of SLPs in conducting aphasia evaluation in Malaysia, which is a country consisting of multiethnic populations, and (b) to determine challenges faced by SLPs when conducting aphasia evaluation involving a diverse group of individuals. Methods: An online survey was distributed to SLPs who are practicing in Malaysia involved in the management of aphasia during the period of data collection. The questionnaire was developed to gather data on participants' background, their practices in evaluating people with aphasia, and challenges that they face related to aphasia evaluation. Proportions were calculated for each item in the questionnaire to determine patterns related to background information, SLP practices and related challenges. Results: Malaysian SLPs were found to gather information about their patients via interviews with caregivers, medical records, and direct testing and observation. Abilities of people with aphasia that were consistently reported to be assessed frequently include auditory language comprehension, verbal expression, repetition of words and sentences, and social communication. Two major challenges identified were linguistic barrier and lack of standardized assessment tools for aphasia evaluation. Conclusions: There are some similarities in terms of SLP practices and challenges faced by the participants in comparison with other studies conducted in diverse contexts. Issues related to cultural and linguistic diversity complicates the development of appropriate resources for aphasia evaluation. Consideration of those issues in development of original and adapted evaluation tools may improve the accuracy of diagnosis, identification of severity, and planning of intervention.
\end{abstract}

\section{Title Page}

Article title: Evaluation of aphasia in a culturally and linguistically diverse context: Practices of and challenges faced by speech-language pathologists

Running title: Evaluation of aphasia in a CLD context (Character count: 38 characters)

Authors and affiliations:

1. Author name (corresponding author): Fatimah Hani Hassan Affiliation: Faculty of Health Sciences, Universiti Kebangsaan Malaysia Email: fatimahhani@ukm.edu.my Postal address: Center for Rehabilitation and Special Needs Studies, Faculty of Health Sciences, Universiti Kebangsaan Malaysia, Jalan Raja Muda Abdul Aziz, 50300 Kuala Lumpur, MALAYSIA. Telephone number: +603-9289-5011 
2. Author name: Pei Whey Heng Affiliation (during study period): Faculty of Health Sciences, Universiti Kebangsaan Malaysia Current affiliation: KPJ Damansara Specialist Hospital

3. Author name: Rahayu Mustaffa Kamal Affiliation (during study period): Faculty of Health Sciences, Universiti Kebangsaan Malaysia Current affiliation: International Islamic University of Malaysia

Funding information: There is no funding received for the study

Conflict of interest statement: All authors declare that there is no conflict of interests associated with the study.

\section{Structured Abstract (word count: 300 words)}

Rationale, aims and objectives: Cultural and linguistic diversity is a major challenge faced by speech-language pathologists (SLPs) in conducting evaluation of aphasia, especially in low-resource regions. Without proper consideration of cultural and linguistic issues when adapting English-based language tests to other languages, performance of individual with aphasia may not be valid and reliable. The present study was conducted: (a) to identify the practices of SLPs in conducting aphasia evaluation in Malaysia, which is a country consisting of multiethnic populations, and (b) to determine challenges faced by SLPs when conducting aphasia evaluation involving a diverse group of individuals.

Methods: An online survey was distributed to SLPs who are practicing in Malaysia involved in the management of aphasia during the period of data collection. The questionnaire was developed to gather data on participants' background, their practices in evaluating people with aphasia, and challenges that they face related to aphasia evaluation. Proportions were calculated for each item in the questionnaire to determine patterns related to background information, SLP practices and related challenges.

Results: Malaysian SLPs were found to gather information about their patients via interviews with caregivers, medical records, and direct testing and observation. Abilities of people with aphasia that were consistently reported to be assessed frequently include auditory language comprehension, verbal expression, repetition of words and sentences, and social communication. Two major challenges identified were linguistic barrier and lack of standardized assessment tools for aphasia evaluation.

Conclusions: There are some similarities in terms of SLP practices and challenges faced by the participants in comparison with other studies conducted in diverse contexts. Issues related to cultural and linguistic diversity complicates the development of appropriate resources for aphasia evaluation. Consideration of those issues in development of original and adapted evaluation tools may improve the accuracy of diagnosis, identification of severity, and planning of intervention.

Keywords: aphasia; speech-language pathologists; evaluation; language test; survey; Malaysia; diversity

Main Text (word count: 4957 words)

\section{Introduction}

\section{Evaluation and Diagnosis of Aphasia}

Aphasia is an acquired language disorder that occurs due to brain injury on the language-dominant hemisphere ${ }^{1}$. A thorough evaluation is crucial in making diagnosis of aphasia, as well as determining appropriate aphasia treatment, including treatment focus, frequency, and length ${ }^{2,3}$. The American Speech-LanguageHearing Association recommended that speech-language pathologists (SLPs) complete a thorough evaluation 
by collecting valid and reliable information from multiple sources, including direct testing, observations, and reports ${ }^{3}$. Following a systematic review of clinical practice guidelines for aphasia rehabilitation, Shrubsole and colleagues proposed (in relation to the evaluation of aphasia) that SLPs: (a) ensure the validity and reliability of assessment tools, (b) collect information that will lead to the development of meaningful and relevant treatment goals, and (c) actively communicate with families, caregivers, co-workers and other healthcare professionals to determine the severity, needs and issues faced by individuals with aphasia in various situations ${ }^{4}$.

Based on the World Health Organization's International Classification of Functioning, Disability and Health Framework (WHO ICF), it was recommended that SLPs focus on multiple facets during aphasia evaluation, such as the individual's background, levels of language capabilities, impacts of communication impairment, and factors that facilitate or impede recovery of aphasia ${ }^{3}$. While it is important to cover all facets of the framework when evaluating people with aphasia (PWA), it is important to note that aphasia is characterized by language skill deficits ${ }^{5}$. PWA has difficulties in comprehending and producing language at various linguistic levels (i.e., lexical-semantic, syntax, and discourse), which may be associated with impaired language processing ${ }^{1}$. Language impairment of aphasia occurs regardless of the modalities used for transmitting or receiving language, including auditory-verbal/spoken, textual, and sign language modes ${ }^{2}$. Due to difficulties in comprehending and producing language in various modalities, communication of PWA with other are negatively affected and requires compensatory support ${ }^{6}$. Language functions may be improved through direct intervention that is based on evaluation findings of language functions ${ }^{7}$.

\section{Language Evaluation in Culturally and Linguistically Diverse Contexts}

When conducting language and communication assessments involving people with diverse background, previous studies have reported that SLPs face challenges in accumulating reliable and valid assessment findings. Impact on reliability and validity of assessment findings may arise due to multiple languages used by the client ${ }^{8}$. In other circumstances, clinicians may be unable to speak languages and/or appreciate beliefs, customary practices and cultural etiquette of clients and their families ${ }^{9}$. These limitations may result in biased judgment that may lead to inappropriate treatment programs for individuals with communication disorders ${ }^{10}$.

Malaysian population consists of multiple ethnic groups, including Malays, indigenous people, Chinese, Indians, and Eurasians ${ }^{11}$. Malays and indigenous people have occupied the Malaysian regions for centuries, while those with Chinese, Indian, and Eurasian backgrounds are the descendants of immigrants during the colonial period ${ }^{12}$. Since the independence of the nation, the Malay language has been employed as the national language and formally taught in schools; however, this language is mainly used for formal government-related affairs and for communication among the Malays ${ }^{13}$. Variations of Chinese dialects, Tamil, English and more than 80 indigenous languages are continued to be used throughout the nation by various ethnic groups ${ }^{13,14}$. In addition, various ethnic groups preserve their unique customs and etiquettes 15. Interestingly, some values are being shared across cultures due to co-existence of different ethnicities for many years ${ }^{16}$.

Due to cultural and linguistic diversity in Malaysia, SLPs are likely to experience difficulties in ensuring the validity of finding from language and communication evaluations. Previous research demonstrated a lack of psychometrically sound assessment tools in Malaysian local languages ${ }^{17,18}$. A lack of appropriate tools for local populations was reported to be a common challenge across various disorders ${ }^{19}$. To reduce the dearth of resources for aphasia evaluation, Van Dort and colleagues adapted the Boston Naming Test into the Malay language; however, in the adapted Malay version, only $48.3 \%$ of the original items were found to be culturally appropriate for the Malaysian Malay population ${ }^{20}$. Other items were found to be less relevant to Malaysian experiences (e.g., pretzel, acorn, and wreath).

In order to improve the present situation, we aim to uncover general practices of Malaysian SLPs in conducting evaluation of aphasia within the Malaysian contexts, in terms of the components of aphasia evaluation, 
methods to gather language-based data, and contexts in which aphasia evaluation was conducted. In addition, we aim to determine the extent to which diversity-related challenges act as a barrier for successful evaluation of aphasia.

\section{Methods}

\section{Study design}

A cross-sectional online survey was employed. The survey consisted of multi-choice items, with an open-ended section for participants to add responses that were not listed in the presented options.

\section{Participants}

An invitation email was sent to the members of Malaysian Association of Speech and Hearing and Malaysian Speech-Language Therapist Association. Participants received background and explanation about the study through the email. An online link of the survey was extended to potential participants who replied to the invitation email and indicated their involvement in aphasia management. All participants met the following inclusion criteria: (a) having a qualification to practice as an SLP in Malaysia and (b) being involved in clinical management of aphasia during data collection period. Participants were requested to provided online consent before completing an online questionnaire.

\section{Materials}

An online questionnaire was developed for the study based on previous studies that focus on SLP practices (Joginder Singh et al . 2011; Mustaffa Kamal et al . 2012; Simmons et al . 2003; Tayloret al . 2009). Three SLPs who primarily focus their clinical work on aphasia management were involved as panel experts for evaluation face and content validity, as well as overall presentation of the survey. Based on their recommendation, we included additional questions and modified sentence structure and item sequence. The questionnaire consisted of three sections as shown in Table 1.

[Table 1]

\section{Data analyses}

Percentages were obtained for categorical data in all sections of the questionnaire. For Section II, percentages were calculated based on Likert scale points. Five points on the Likert scale were recategorized into three levels of frequency: "Never" for the first point, "Less Frequent" for the second and third points, and "More Frequent" for the fourth and fifth points. Three levels of consistency (Highly Consistent, Moderately Consistent, and Inconsistent) were also determined for each level of frequency of assessment activities. A specific aspect or activity was considered to be Highly Consistent (HC) when at least $80 \%$ of participants agree on considering an aspect or conducting an activity at the same frequency level. An agreement level of $50-79 \%$ on a specific aspect or activity was considered to be Moderately Consistent (MC). Inconsistent (IC) level was considered for an agreement level that was less than 50\% (Trochim and Donelly 2007, Mustaffa Kamal et al . 2012). In addition, nine types of challenges were presented to participants. The proportions of participants who reportedly have been facing specified challenges were calculated. 


\section{Results}

\section{Participants' demographic information}

The online survey was distributed to 107 Malaysian SLPs who fit the inclusion criteria. A total of 32 participants completed the survey, which resulted in a response rate of $29.9 \%$. Participants' demographic information is summarized in Table 2 .

[Table 2]

Frequency and consistency of aphasia assessment aspects and activities. The level of frequency and consistency were identified for the following aspects and activities: (a) documentation of case history and background information, (b) components of aphasia assessment, (c) methods of language and communication skills evaluation, and (d) contexts of aphasia evaluation. Table 3 summarizes the frequency and consistency levels for each aspect or activity. Practices that were to be conducted frequently and consistently (rated by more than $80 \%$ of the participants) are:

1. Documentation of case history via interview and medical records

2. Evaluation of auditory language comprehension and spoken language/verbal expression

3. Evaluation of repetition of words and sentences

4. Evaluation of pragmatic and social skills

5. Unstructured observation of language and communication functions

6. Application of informal assessment approach

7. Evaluation of aphasia in clinical settings

[Table 3]

Challenges in aphasia assessment. The challenges were grouped into three categories, which are barriers related to cultural-linguistic diversity, limitation of support and resources, and limitation of clinical competency among SLPs in aphasia evaluation. The proportion for each challenge as rated by the participants is shown in Table 4. Two types of challenges were identified to be experiences by more than half of the participants, which include: (a) language differences between PWA and clinicians, and (b) lack of standardized tool for aphasia evaluation.

[Table 4]

\section{Discussion}

\section{Aphasia Evaluation: Practices of Malaysian SLPs}

In the present study, we revealed practices of SLPs and related issues pertaining to the evaluation of aphasia. In general, it was found that SLPs reported consistency of practices in certain aspects of aphasia evaluation. Four trends were identified related to their aphasia evaluation practices.

Firstly, SLPs were found to consistently obtain case history and background information through patient/caregiver interviews and medical records. As mentioned earlier, Shrubsole and colleagues recommended for the involvement and collaboration of SLPs with PWA and individuals who are relevant in their intervention. Involvement of family and caregivers in speech-language management of aphasia is crucial to ensure positive intervention outcomes following carry-over of SLPs recommendation in home environment ${ }^{21}$. The results of this study may indicate that Malaysian SLPs are aware of the importance of family involvement in successful aphasia care. Unlike family involvement, collaboration of SLPs with other healthcare professionals was less consistent. Malaysian SLPs seemed to rely more on medical records as compared to direct engagement with other healthcare professionals. Direct communication with professionals from other disciplines may support in-depth understanding of patients' conditions, roles of SLPs in aphasia intervention, and gain 
perspectives of the professionals regarding needs, facilitators and barriers that may impact aphasia recovery 22. It is important to note that most of the participants worked in acute public hospitals. In this type of setting, SLPs and non-SLP professionals tend to have heavy caseloads and might only confer on select cases 23.

Secondly, SLPs were found to focus their evaluation on auditory language comprehension, spoken language/verbal expression, repetition of words and sentences, and pragmatic/social communication skills. Since aphasia is affecting the ability of PWA to understand, produce, and use language for communication ${ }^{2}$, it was expected that SLPs direct their evaluation on those abilities. Across the evaluation components rated by the participants, all of them unanimously reported that pragmatic and social communication skills are consistently evaluated. Findings from pragmatic and social communication assessments may inform SLPs regarding competency of PWA to use language when communicating with others ${ }^{24}$. Although reading comprehension and written expression abilities are also defining characteristics of aphasia ${ }^{2}$, it is surprising that SLPs demonstrated lower consistency in evaluating those abilities. Lack of consistency in evaluating reading and writing abilities may be related to the limited available time for SLPs to complete assessments during the initial session. In other countries, limited time with individual patients have reportedly been a challenge for SLPs, not only in assessing PWA, but also in providing treatment ${ }^{25-27}$. While initial evaluation findings are useful for determining the presence and severity of aphasia, continuous evaluations must be conducted to ensure provision of appropriate treatment based on the needs of individuals with aphasia ${ }^{2,28}$. Language and communication abilities among people with aphasia (PWA) have been proven to change with time ${ }^{29}$. Continuous assessment of aphasia also may address components that have not been thoroughly evaluated during the initial session.

Thirdly, SLPs were found to rely upon unstructured and informal means to evaluate PWAs' language and communication abilities. Unlike the practices of SLPs in Singapore (a neighboring country), the participants reported moderate consistency in utilizing formal assessment tools, which primarily aim to determine the skills that are impaired and the extent of impairment. In the Singaporean study, more than $90 \%$ of their SLP participants utilized an assessment tools for evaluation of aphasia ${ }^{30}$. In the present study, only about $50 \%$ of the participants have been using formal assessment tools frequently, while the remaining half either use the tools infrequently or not at all. This may be due to the lack of standardized tools can be accessed by SLPs, which has been identified by the participants as a common challenge. Three tools were found to be used in both Singapore and Malaysia, which are the Boston Diagnostic Aphasia Examination (BDAE) ${ }^{31}$, Psycholinguistic Assessments of Language Processing in Aphasia (PALPA) ${ }^{32}$ and Comprehensive Aphasia Test (CAT) ${ }^{33}$. Although these tools are more often used in both countries, SLPs utilized those tools minimally. More consistently, Malaysian SLPs have been employing informal approach to gather evaluation findings.

Finally, evaluation of aphasia was found to be primarily limited to clinical settings. This may be due to nature of service provision at SLPs' workplaces. More than $80 \%$ of study participants reported to work in acute hospitals, which is a trend that has been reported in other studies ${ }^{17,18,34,35}$. According to Ahmad and colleague, the Malaysian Ministry of Health has been a primary source of employment for graduates of local SLP university programs, thus, many SLPs are working in public hospitals through the country ${ }^{19}$. SLPs in acute public hospitals tend to serve individuals with communication and/or swallowing impairments across the lifespan, which result in high caseloads and limited time to engage with PWA in non-clinical settings

34. Since aphasia affects communication abilities of PWA, it has been noted that aphasia evaluation that is conducted in social and personally-relevant environment, such as their workplace and personal homes, may provide critical information for designing an effective treatment $\operatorname{program}^{36}$.

\section{Issues on Cultural and Linguistic Diversity in Aphasia Evaluation}

Across the nine challenges presented in the survey, linguistic barriers and lack of standardized resources were rated to be more common among SLPs. As mentioned earlier, the Malaysian population consists of diverse ethnic groups, who are using a variety of languages and/or dialects. A huge majority of Malaysians are able 
to communicate in more than one language ${ }^{13,14}$. In the case of aphasia, proficiency of one or more language may be affected differently, hence the need to evaluate functioning of all languages used by PWA prior to the onset of aphasia ${ }^{37,38}$. Varied effects of language impairment on PWAs' abilities in different languages may be due to the extent in which one language is better preserved than another ${ }^{37}$. In addition, the age when one acquire the second and additional languages have been found to influence aphasia recovery for that particular language ${ }^{39}$.

It can also be inferred that those two challenges (i.e., linguistic barrier and lack of standardized language tools) are related. In conducting language evaluation, SLPs commonly translate and adapt assessment tools that has been developed and normed based on English-speaking population ${ }^{30,37,40}$. The complexity of the processes involved in translating and adapting standardized English language tools is well acknowledged 41. Clinicians are required to consider various attributes associated with diverse cultures, linguistic features (including semantics, grammaticality, and syntactic structures) and factors that may influence performance level in those test, such as item familiarity, word frequency, and age of word acquisition ${ }^{40,42}$. Because linguistic and culture-related aspects must be considered in order to adapt English tools to local languages, the progress of resource development for aphasia evaluation tend to be slow, thus, affecting the availability of standardized language tools for local populations. Additionally, lack of expertise and financial support may also restrict the progress in developing tools for aphasia evaluation ${ }^{19,43}$.

Experts have suggested strategies to address these challenges. One of the strategies involve the adaptation of tools that are generally neutral in terms of the stimuli used in testing ${ }^{44}$. For example, the Revised Token Test (RTT) ${ }^{45}$ primarily includes basic colors, prepositions, and shapes for its language stimuli. It is important to note that this strategy may not address all issues related to cultural and linguistic diversity. In adapting the RTT into standard Indonesian language, Jap and Arumsari found that the usability of the adapted version is limited for individuals who are using various other Indonesian dialects ${ }^{46}$. Therefore, assessment findings based on the adapted tests must be treated cautiously.

Another strategy for addressing assessment of aphasia with multilingual and culturally diverse population is related to the discourse analysis approach, where language and communication samples are collected by SLPs within and outside of clinical contexts ${ }^{47}$. Discourse analysis was found to provide SLPs with accurate diagnosis of aphasia, as well as its types and severity ${ }^{48}$. However, discourse analysis requires training for SLPs to acquire specific skill set in eliciting language and communication samples and analyze content and linguistic characteristics accurately ${ }^{49}$. Bryant and colleagues found that even among SLPs who have been trained to conduct discourse analysis, many did not apply the method due to time constraint at their workplace ${ }^{49}$.

Although cultural and linguistic issues related to aphasia evaluation cannot be resolved immediately, strategies suggested by previous researchers and experts can be applied with proper consideration. At the same time, effort to develop assessment resources for diverse populations must be enhanced and supported in order to speed up the process. Building professional network may also encourage support among SLPs via sharing resources, experience and methods that may be applied relevant to the local contexts.

\section{Limitation of the Study.}

The present study only focused on evaluation of language functions in aphasia assessment and challenges faced by SLPs related to it. Focus on other aspects of evaluation was minimal. This study did not include the practices of SLPs in the evaluation of access to communication for PWA and impacts of aphasia on the lives of PWA. Future studies are needed for discovering the frequency and extent of evaluation processes in addressing all facets proposed in WHO ICF ${ }^{50}$. Based on the ICF, evaluation of aphasia must also include impacts of communication impairment on the lives of PWA, as well as factors that may affect aphasia recovery and opportunities for PWA to participate in social and personally relevant activities, including management of personal finances, involvement in hobbies before the onset of aphasia, and recommencement of roles prior to aphasia in the context of family and community. By looking at various psychosocial aspects, cultural 
impacts on evaluation practices might be more visible. Participation of PWA and their caregivers may also give better insights for improving guidelines and protocols for aphasia evaluation.

\section{References}

1. McNeil MR, Pratt SR. Defining aphasia: Some theoretical and clinical implications of operating from a formal definition. Aphasiology . 2001;15(10/11):901-911.

2. Hallowell B. Aphasia and Other Acquired Neurogenic Language Disorders . Plural Publishing Group; 2017.

3. American Speech-Language-Hearing Association. Aphasia: Assessment. ASHA: American Speech-Language-Hearing Association. Published October 13, 2017. Accessed September 11, 2019. https://www.asha.org/PRPSpecificTopic.aspx?folderid=8589934663\&section=Assessment

4. Shrubsole K, Worrall L, Power E, O'Connor D. Recommendations for post-stroke aphasia rehabilitation: An updated systematic review and evaluation of clinical practice guidelines. Aphasiology . 2017;31:1-24. doi: $10.1080 / 02687038.2016 .1143083$

5. Tippett DC, Hillis AE. Where are aphasia theory and management "headed"? F1000Research . 2017;6. doi:10.12688/f1000research.11122.1

6. Johansson MB, Carlsson M, Sonnander K. Communication difficulties and the use of communication strategies: From the perspective of individuals with aphasia. Int J Lang Commun Disord . 2012;47(2):144155. doi:10.1111/j.1460-6984.2011.00089.x

7. Brady MC, Kelly H, Godwin J, Enderby P, Campbell P. Speech and Language Therapy for Aphasia Following Stroke. The Cochrane Collaboration; 2016. Accessed June 14, 2016. http://onlinelibrary.wiley.com/doi/10.1002/14651858.CD000425.pub4/abstract

8. White CDL, Jin L. Evaluation of speech and language assessment approaches with bilingual children. Int J Lang Commun Disord . 2011;46(6):613-627. doi:10.1111/j.1460-6984.2011.00049.x

9. Mahendra N. Advancing inclusion and social justice in aphasia care: Three things you can do. In: ; 2019.

10. Centeno JG. Guest Editor's Column: Clinical Management of Communicatively-Impaired Adult Neurorehabilitation Caseloads in a Diverse Aging World. Perspect ASHA Spec Interest Groups . 2017;2(2):88-90. doi:10.1044/persp2.SIG2.88

11. Department of Statistics Malaysia. Current population estimates, Malaysia, 2017-2018. Department of Statistics Malaysia Official Portal. Published July 31, 2018. Accessed December 19, 2018. https://www.dosm.gov.my/v1/index.php?r=column/cthemeByCat\&cat=155\&bul_id=c1pqTnFjb29HSnNYNUpiTmNWZHArdz09\&menu_id=L0pheU43NWJwRWVSZklWdzQ4TlhUUT09

12. Kok KC. Modern Certificate Guides: History of Malaya . Oxford University Press; 1978.

13. Smith KJ. Minority language education in Malaysia: Four ethnic communities' experiences. Int J Biling Educ Biling . 2003;6(1):52-65. doi:10.1080/13670050308667772

14. Tan YS, Teoh HS. Ethnic contestation and language policy in a plural society: The Chinese language movement in Malaysia, 1952-1967. Hist Educ . 2014;43(2):251-268. doi:10.1080/0046760X.2014.880751

15. Munan H. Culture Shock! A Guide to Customs and Etiquette . Times Books International; 2001.

16. Wan Muhammad WR. Adat Resam Masyarakat Malaysia. Jabatan Kebudayaan dan Kesenian Negara; 2006 . 
17. Joginder Singh S, Chan MY, Ahmad Rusli Y. Practise patterns of Malaysian speech-language pathologists in managing children with speech and language delay/disorder. Int J Speech Lang Pathol . 2016;18(6):560570. doi:10.3109/17549507.2016.1139624

18. Phoon HS, Maclagan M. A survey of Malaysian speech-language pathologists' perception of articulation and phonological assessments. Asia Pac J Speech Lang Hear . 2009;12(4):315-332.

19. Ahmad K, Ibrahim H, Othman BF, Vong E. Addressing education of speech-language pathologists in the World Report on Disability: Development of a speech-language pathology program in Malaysia.Int $J$ Speech Lang Pathol . 2013;15(1):37-41. doi:10.3109/17549507.2012.757709

20. Van Dort S, Vong E, A Razak R, Mustaffa Kamal R, Hooi PM. Normative data on a Malay version of the Boston Naming Test. J Sains Kesihat Malays . 2007;5(1):27-36.

21. Hallé M, Le Dorze G, Mingant A. Speech-language therapists' process of including significant others in aphasia rehabilitation. Int J Lang Commun Disord . 2014;49(6):748-760. doi:10.1111/1460-6984.12108

22. Cardinal LA, Freeman-Sanderson A, Togher L. The speech pathology workforce in intensive care units: Results from a national survey.Aust Crit Care . 2020;33(3):250-258. doi:10.1016/j.aucc.2020.02.003

23. Horton S, Lane K, Shiggins C. Supporting communication for people with aphasia in stroke rehabilitation: Transfer of training in a multidisciplinary stroke team. Aphasiology . 2016;30(5):629-656. doi:10.1080/02687038.2014.1000819

24. Sobhani-Rad D. A review on adult pragmatic assessments. Iran J Neurol . 2014;13(3):113-118.

25. Rose M 1, Attard MC. Practices and challenges in community aphasia groups in Australia: Results of a national survey. Int J Speech Lang Pathol . 2015;17(3):241-251. doi:10.3109/17549507.2015.1010582

26. Tiwari S, Krishnan G. Aphasia rehabilitation in India: A preliminary survey of speech-language pathologists. J India Inst Speech Hear . 2011;30:108-116.

27. Klippi A, Sellman J, Heikkinen P, Laine M. Current clinical practices in aphasia therapy in Finland: Challenges in moving towards national best practice. Folia Phoniatr Logop . 2012;64:169-178. doi:10.1159/000341106

28. Patterson JP, Chapey R. Assessment of language disorders in adults. In: Chapey R, ed. Language Intervention Strategies in Aphasia and Related Neurogenic Communication Disorders . 5th ed. Lippincott Williams \& Wilkins; 2008:64-160.

29. Holland A, Fromm D, Forbes M, MacWhinney B. Long-term recovery in stroke accompanied by aphasia: A reconsideration. Aphasiology . 2017;31(2):152-165. doi:10.1080/02687038.2016.1184221

30. Guo E, Togher L. Speech pathology services for peoplewith aphasia: What is the current practice in Singapore? Disabil Rehabil . 2013;36(8):691-704. doi:10.3109/09638288.2013.804597

31. Goodglass H, Kaplan E, Barresi B. Assessment of Aphasia and Related Disorders . 3rd ed. PAR, Inc.; 2001.

32. Kay J, Lesser R, Coltheart M. PALPA: Psycholinguistic Assessments of Language Processing in Aphasia . Lawrence Erlbaum Associates; 1992.

33. Swinburn K, Porter G, Howard D. Comprehensive Aphasia Test . Psychology Press; 2004.

34. Diong ZZ, Rose T, Scarinci N, Power E, Siyambalapitiya S. Speech-language pathology services for adults with post-stroke aphasia in Malaysia. In: ; 2019.

35. Mustaffa Kamal R, Ward EC, Cornwell P, Sharma S. Provision of dysphagia services in a developing nation: Infrastructural challenges.Int J Speech Lang Pathol . Published online April 15, 2015:1-11. 
36. Pommerehn J, Delboni MCC, Fedosse E. International Classification of Functioning, Disability and Health, and aphasia: A study of social participation. Soc Bras Fonoaudiol . 2016;28(2):132-140. doi:10.1590/2317-1782/201620150102

37. Paradis M. Principles underlying the Bilingual Aphasia Test (BAT) and its uses. Clin Linguist Phon . 2011;25(6-7):427-443.

38. Paradis M. Bilingualism and aphasia. In: Whitaker H, Whitaker H, eds. Studies in Neurolinguistics . Vol 3. Academic Press; 1977:65-121.

39. Kuzmina E, Weekes BS. Role of cognitive control in language deficits in different types of aphasia. Aphasiology . 2017;31(7):765-792. doi:10.1080/02687038.2016.1263383

40. Centeno JG. Assessing services with communicatively impaired bilingual adults in culturally and linguistically diverse neurorehabilitation programs. $J$ Commun Disord . 2015;58:58-73. doi:10.1016/j.jcomdis.2015.10.005

41. Ivanova MV, Hallowell B. A tutorial on aphasia test development in any language: Key substantive and psychometric considerations.Aphasiology . 2013;27(8):891-920. doi:10.1080/02687038.2013.805728

42. Heuer S, Hallowell B. An evaluation of multiple-choice test images for comprehension assessment in aphasia. Aphasiology . 2007;21(9):883-900. doi:10.1080/02687030600695194

43. A Aziz MA. Aphasia research in Malaysia: Where are we now? Presented at the: Aphasia and linguistic network in Malaysia and Indonesia; August 24, 2019; Putrajaya.

44. Turkyilmaz MD, Belgin E. Reliability, validity, and adaptation of Computerized Revised Token Test in normal subjects. J Int Adv Otol . 2012;8(1):103-112.

45. McNeil MR, Prescott TE. Revised Token Test . pro-ed; 1978.

46. Jap BAJ, Arumsari C. Adaptation of the Token Test in standard Indonesian. Makara Hubs-Asia . 2017;21(1):44-51. doi:10.7454/mssh.v2lil.3499

47. Kong APH. Aphasia assessment in Chinese speakers. ASHA Lead . 2011;16(13):36-38. doi:10.1044/leader.FTR8.16132011.36

48. Kong APH, Wong CW-Y. An integrative analysis of spontaneous storytelling discourse in aphasia: Relationship with listeners' rating and prediction of severity and fluency status of aphasia. Am J Speech Lang Pathol . 2018;27(4):1491-1505. doi:10.1044/2018_AJSLP-18-0015

49. Bryant L, Ferguson A, Valentine M, Spencer E. Implementation of discourse analysis in aphasia: Investigating the feasibility of a Knowledge-to-Action intervention. Aphasiology . 2019;33(1):31-57.

50. World Health Organization. WHO | International Classification of Functioning, Disability and Health (ICF). WHO. Published 2001. Accessed November 28, 2017. http://www.who.int/classifications/icf/en/

\section{Acknowledgement}

We thank Universiti Kebangsaan Malaysia for providing physical support to conduct this study. We are grateful for the feedback given by faculty members of the UKM Speech Sciences Programme to improve the quality of the student. Finally, we extend our appreciate to all participants for taking part in the study.

Tables

Table 1: Questionnaire sections and description 


\begin{tabular}{|c|c|c|}
\hline Questionnaire section & Number of items & Description of section \\
\hline $\begin{array}{l}\text { Section I: Participants' } \\
\text { background }\end{array}$ & 6 & $\begin{array}{l}\text { This section consists of } \\
\text { multiple-choice questions to } \\
\text { obtain participants' background } \\
\text { information, including academic } \\
\text { qualification, years of } \\
\text { experience working as an SLP, } \\
\text { types of clinical settings, and } \\
\text { clinical workload. }\end{array}$ \\
\hline $\begin{array}{l}\text { Section II: Aphasia assessment } \\
\text { practice }\end{array}$ & 57 & $\begin{array}{l}\text { This section consists of 5-point } \\
\text { Likert scale items that cover } \\
\text { various aspects and activities } \\
\text { related to aphasia evaluation: } \\
\text { Documentation of case history } \\
\text { and background information } \\
\text { Components of language and } \\
\text { communication assessment } \\
\text { Evaluation methods Contexts of } \\
\text { evaluation }\end{array}$ \\
\hline $\begin{array}{l}\text { Section III: Challenges faced by } \\
\text { SLPs }\end{array}$ & 3 & $\begin{array}{l}\text { This section consists of } \\
\text { multiple-choice questions on } \\
\text { challenges related to linguistic } \\
\text { and cultural diversity, resource } \\
\text { limitation and competency } \\
\text { level. }\end{array}$ \\
\hline
\end{tabular}

Table 2: Demographic information of participants

\begin{tabular}{lc}
\hline Demographic information & $\%(n)$ \\
\hline $\begin{array}{l}\text { Highest academic qualification Bachelor's degree (local university) } \\
\text { Master's degree (abroad university) }\end{array}$ & $96.9 \%(31) 3.1 \%(1)$ \\
Years of working experience Less than 3 years & $56.3 \%(18) 31.2 \%(10) 12.5 \%(4)$ \\
3 to 10 years & \\
More than 10 years & $84.4 \%(27) 15.6 \%(5)$ \\
Clinical setting Acute hospital (public and private sectors) \\
$\begin{array}{l}\text { Community-based clinic (non-profit organization and university settings) } \\
\text { Aphasia workload (of total workload) Less than 25\% }\end{array}$ \\
$\begin{array}{l}25 \% \text { to } 75 \% \\
\text { More than } 75 \%\end{array}$ \\
\hline
\end{tabular}

Table 3: Level of frequency and consistency of aphasia assessment aspects and activities

Assessment aspect and activity

Documentation of case history and background information PWA and caregiver interviews Medical records Interprofessiona Components of aphasia assessment Auditory language comprehension Identifying spoken single words Following simple inst Table 3 (continued)

Methods of language and communication skills evaluation Unstructured assessment Collection of language samples Unstruc 
Assessment aspect and activity

Table 3 (continued)

Contexts of aphasia evaluation Clinical setting (hospital/center) Social activities Home/Personal living space

Table 4: Percentages of participants according to specific challenges in aphasia management

Challenge in aphasia management

$\%(n)$

Cultural-linguistic barriers Differences of dominant language of PWA versus clinicians

$62.5 \%(20) 37.5 \%(12)$

Differences of cultural values of PWA versus clinicians

Support and resource limitations Lack of appropriate infrastructure

$15.6 \%$ (5) $62.5 \%(20) 21.9 \%(7) 9.4$

Lack of standardized assessment tools

Limited access to continuing education resources

Limited access to trained translators

Clinical competency limitations Lack of knowledge on updated information

Lack of clinical skills

$50.0 \%(16) 37.5 \%(12) 34.4 \%(11)$

Lack of confidence 